\title{
ESTIMACIÓN DE LA TASA DE INFESTACIÓN DE LA BROCA EN UN CAFETAL, UTILIZANDO UN MODELO TIPO SIR
}

\section{ESTIMATION OF THE INFESTATION RATE IN \\ COFFEE BERRY BORER, USING A SIR MODEL}

\author{
MÓNICA J. MeSA* OSCAR E. MOLInA ${ }^{\dagger}$
}

Alejandra M. PUlgarín ${ }^{\ddagger}$

Received: 21/Jul/2016; Revised: 18/Apr/2017;

Accepted: 23/Apr/2017

\begin{abstract}
Revista de Matemática: Teoría y Aplicaciones is licensed under a Creative Commons Reconocimiento-NoComercial-Compartirigual 4.0 International License.

Creado a partir de la obra en http://www.revistas.ucr.ac.cr/index.php/matematica

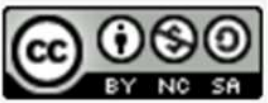

* Doctora en Ingeniería, Facultad de Educación, Universidad del Quindío, Armenia, Colombia. E-Mail:mjmesa@uniquindio.edu.co

${ }^{\dagger}$ Magister en Biomatemáticas, Facultad de Educación, Universidad del Quindío, Armenia, Colombia. E-Mail: omolina@uniquindio.edu.co

${ }^{\ddagger}$ Misma dirección que/Same address as: O.E. Molina. E-Mail: ampulgarin@uniquindio.edu.co
\end{abstract}




\title{
Resumen
}

La broca del café Hypothenemus hampei, es una de las plagas con mayor incidencia y afectación en los cafetales. Colombia, por ser una región que favorece al crecimiento e infestación de esta plaga debido a factores como el clima, ubicación geográfica, producción continua de café, y dado que la caficultura es una de las actividades agrícolas más importantes en el país, es importante realizar estudios referentes a la dinámica de la broca.

Por tal razón, en este artículo se adaptó el comportamiento de la broca del café, con un modelo epidemiológico tipo SIR propuesto por Kermack y Mckendrick. Además usando la relación de tamaño final (propuesta por Castillo Chavez) se estimó el coeficiente que representa la infestación de la plaga en términos de otros parámetros involucrados en la dinámica. Por último, se realizaron diferentes simulaciones numéricas en ambiente Matlab variando los valores de los parámetros del sistema dinámico.

Palabras clave: broca del café; modelado matemático; modelo tipo SIR; estimación de parámetros.

\begin{abstract}
The coffee berry borer, Hypothenemus hampei, is one of the plagues with the highest incidence and affectation in the coffee plantation. Colombia is a region that promotes the growth and infestation of this plague. The infestation is due to several factors such as Colombia's climate, geographic location and continuous coffee production. Coffee production is one of the most important agricultural activities in the country. Therefore, it is important to carry out studies concerning the dynamics of the coffee borer. For this reason, this article adapts the behavior of the coffee borer with an epidemiological model type SIR proposed by Kermack y Mckendrick. This model and the final size relation (proposed by Castillo Chavez) will be used to estimate the coefficient that represents the infestation of the plague in terms of the other parameters involved in the dynamic. Besides, different numerical simulations were done in Matlab environment by changing the values of the parameters of the dynamic system.
\end{abstract}

Keywords: coffee berry borer; mathematical modeling; type model SIR; parameter estimation.

Mathematics Subject Classification: 34D23, 93D20, 65L05.

\section{Introducción}

La broca del café Hypothenemus hampei, es una de las plagas más severas para este cultivo, y se encuentra presente en la mayoría de las regiones productoras 
del grano en el mundo. La broca hace daño al atacar la cereza y reproducirse internamente en el endospermo causando la pérdida parcial o total del grano, y en muchos casos, la caída prematura de los granos [9].

Cuando la broca llega a un sitio por primera vez, desarrolla todo su potencial biótico para alcanzar altas poblaciones en los cafetales que invade. Se ve favorecida por diferentes factores tales como: producción continua de café debido a las condiciones propicias del clima, altas densidades de siembra, lugares con topografía de díficil acceso para realizar las prácticas de control, entre otras [13].

La caficultura es una de las actividades agrarias más importantes en Colombia, debido entre otras cosas, a las divisas que genera, por lo que resulta indudable que cualquier problema fitosanitario que ponga en riesgo la producción y calidad de la cosecha, representa una seria amenaza para la economía regional y nacional, pues de la producción de café como industria, dependen un alto número de familias y labriegos. De acuerdo con lo anterior y teniendo en cuenta que la broca del café es la principal problemática a la que se enfrentan los caficultores a nivel mundial, los estudios que se hagan alrededor de esta plaga, van a redundar en beneficio para la sociedad.

En las condiciones climáticas y geográficas de la zona central cafetera, se ha podido establecer que en un fruto de café, desde el momento en que éste es susceptible al ataque de la plaga hasta la época de cosecha, se pueden producir dos generaciones del insecto. Pero si el fruto no es recolectado, se pueden presentar hasta cuatro generaciones [12].

El uso de insecticidas para el control de la broca del café no es recomendable ya que cuando el insecto ha alcanzado el interior del fruto, este tipo de control no tiene efecto debido a que este actua por contacto. El control químico debe entonces usarse solo cuando la broca se encuentra en el proceso de penetración de los nuevos frutos. Esta situación hace aún más dificultuosa la práctica de control químico en los cafetales, ya que en nuestro país se presentan múltiples floraciones [7], tanto así que en algunas zonas del país, es posible encontrar durante todo un año frutos susceptibles de ser atacados por el insecto [1, 14].

Por estas razones, es importante realizar estudios alrededor de la dinámica de la broca del café y desarrollar trabajos que busquen tener más información de la plaga, con el fin de contribuir a mejorar el manejo de ésta y lograr una mayor productividad del grano.

Lo que se busca con este artículo es modelar la infestación de la broca por medio de un modelo de uso epidemiologico tipo SIR de Kermack y McKendrick y poder estimar el coeficiente de infestación de un cafetal por la plaga de la broca. Inicialmente en la sección 2 se hará una pequeña descripción de la biología de la broca. En la sección 3 se presenta el modelo matemático: los supuestos, 
variables y parámetros, el sistema de ecuaciones que representa la dinámica y puntos de equilibrio. La sección 4 se centra en la estimación del parámetro mencionado anteriormente. En la sección 5 se hace un análisis cualitativo del modelo, al igual que las simulaciones númericas, las cuales permitirán visualizar y analizar la dinámica del modelo que representa el comportamiento de la broca del café. Por último, en la sección 6 se dan unas conclusiones obtenidas del trabajo realizado.

\section{Biología de la broca del café}

Respecto a la biología de la broca del café, se sabe que es de color negro, muy pequeña, de apariencia similar a los gorgojos. Es un insecto holometábolo, lo cual significa que las etapas de su ciclo de vida son: huevo, larva, prepupa, pupa y el estado adulto [13]. Ciclo que se describe brevemente de la siguiente manera: el adulto hembra $H$. hampei una vez emerge, tarda 4 días para estar listo para la reproducción; su periodo de ovoposición es de unos 20 días y coloca entre 2 y 3 huevos por día. En promedio una hembra puede ovipositar 74 huevos durante su vida. La incubación del huevo dura 7,6 días (a 23 grados centígrados) y el estado de larva 15 días para los machos y 19 días para las hembras, la prepupa 2 días y la pupa 6,4 días (a 25,8 grados centígrados). El ciclo total del huevo a emergencia de adultos se estima en 27,5 días (a 24,5 grados centígrados).

La broca del café, es una plaga que inicia su ataque en los frutos verdes del cafeto, entre los 3 y 4 meses después de la floración, cuando estos se encuentran en estado leñoso o pastoso. En la medida en que se permite un mayor ataque en frutos verdes, mayor será el daño en los granos pintones, maduros y sobremaduros. Se ha observado que si se omiten controles, puede llegar a infestar todos los frutos de un cafetal (100\% de infestación) o sea que su crecimiento sólo se ve limitado por la disponibilidad de frutos que se encuentran en los cafetos [9].

Al registrar el tiempo que una hembra demora en penetrar un fruto, se encontró que este varía dependiendo del estado de desarrollo del fruto de la siguiente manera: en frutos verdes 5 horas y 36 minutos, en frutos pintones 5 horas y 54 minutos, en frutos maduros 4 horas y 50 minutos y en frutos secos 11 horas y 21 minutos [10].

Comúnmente los frutos del café empiezan a ser susceptibles al ataque de la broca cuando alcanzan un peso seco mayor o igual al $27 \%$, lo cual se logra cuando el fruto alcanza más de 150 días de desarrollo (dependiendo del lugar geográfico), es decir, que el periodo de vulnerabilidad de la broca a un insecticida de contacto se concentra entre 120 y 150 días después de la floración [12]. 
Cuando el insecto inicia el ataque sobre frutos no muy desarrollados (menor a 150 días), el tiempo de exposición en el canal de penetración es muy prolongado, ya que debe esperar a que la consistencia de las almendras sea adecuado para iniciar su ovíposición, volviéndola vulnerable al tratamiento con insecticidas químicos y biolológicos durante este tiempo.

La dispersión de la broca del café se inicia con el abandono del fruto de origen por parte de la hembra y termina con la respuesta a los estímulos del hospedero, que en este caso es el grano de café.. Los machos son ápteros y permanecen toda su vida dentro del fruto de origen. El hecho de que las hembras abandonen el fruto de origen se conoce como comportamiento de abandono. Las hembras han sido fecundadas antes de abandonar el fruto donde emergieron $\mathrm{y}$ poseen alas funcionales, salen del fruto por efecto de las lluvias o la alta insolación.

Durante el periodo de crecimiento del fruto, las hembras emergen de manera continua en busca de nuevos frutos. Casi siempre, al finalizar la cosecha (se ha visto principalmente en centroamérica), se presentan migraciones masivas de la plaga debido a la poca oferta de frutos en este periodo. Sin embargo, gran parte de ellas permanecen en los frutos sobremaduros que no fueron recolectados. En este tipo de frutos, la emergencia y la ovoposición se detienen por completo, concentrándose una gran cantidad de adultos. Este fenómeno es conocido como "diapausa reproductiva". En épocas de lluvia, cuando se induce la nueva floración del cafeto, se rompe esta diapausa, lo que provoca una emergencia masiva de las hembras de los frutos secos. Es de anotar que se han registrado (en estudios de laboratorio) grandes periodos de vuelo de los insectos, que van desde 90 minutos de vuelo ininterrumpido o 180 minutos de manera intermitente [6]. Sin embargo, no todas las hembras emigran, algunas se quedan en el fruto de origen. Aunque la condición necesaria para que una hembra inicie la emergencia del fruto de origen es la fecundación, no todas las que han sido fecundadas salen del fruto debido por causas como la falta de desarrollo alar, carencia de depósitos de grasa (que constituyen la reserva de energía para el vuelo) o una baja propensión para el vuelo.

La selección del nuevo hospedero comprende entre la percepción de los estímulos olfativos provenientes del hospedero hasta el inicio de la alimentación de la hembra. En cuanto al aterrizaje, el proceso podría ser aleatorio [15], en el cual los individuos posarían sobre un hospedero o no hospedero, sin embargo esto puede ser interpretado como una primera aproximación a la zona apropiada y no necesariamente al fruto específico. Bustillo y Villalba 2004 proponen que la broca es inicialmente atraída por el olor, luego por el color y la forma del fruto.

La etapa de la concentración de los escolitinos (insecto de la broca) se da 
una vez que los primeros individuos colonizadores, previa evaluación del sustrato, comienzan a liberar feromonas de agregación que pueden tener un efecto sinérgico con volátiles de la planta, para orientar al resto de la población hacia su lugar de ubicación y generar así una alta concentración de la población en el hospedero [15]. A este proceso se le conoce como atracción secundaria. Las evidencias indican que estas feromonas de agregación podrían encontrarse en las heces de las hembras [6].

La última etapa de la dispersión dela broca se conoce como establecimiento. Esta etapa inicia con la perforación de los frutos, previa evaluación de la calidad del recurso y concluye con la ovoposición.

\section{Modelo matemático}

En esta sección se describe la dinámica de la propagación de la Broca en un cafetal, basado en el modelo epidemiológico SIR (Suceptibles, Infectados y Recuperados), propuesto por Kermack y McKendrick en 1927.

\subsection{Supuestos del modelo}

Para adaptar el modelo de tipo SIR a la dinámica de la propagación de la broca del café, se deben considerar los siguientes supuestos.

1. La población de plantas de café está distribuída homogéneamente en la zona de estudio. De ésta manera todos los cafetos tienen el mismo grado de interacción con la broca, por lo tanto, cada cafeto tendrá la misma probabilidad de ser infestado.

2. Un árbol de café se considera suceptible, si al hacer el muestreo la proporción de granos de café afectados por la plaga en la rama seleccionada es menor del 5\%. Este porcentaje será asumido como el umbral de infestación de una planta de café.

3. Se considera que una planta está infestada si una rama cualquiera tiene un porcentaje de infestación más alto del umbral establecido.

4. La subpoblación de cafetos recuperados, son aquellas plantas que debido a algún control [5], bajan su nivel de infestación por debajo del umbral establecido.

5. La población de plantas de café es constante, es decir, no hay nuevos individuos ni tampoco se excluyen plantas de café. En el modelo no se 
consideran efectos demográficos en la población, esto significa que la escala de tiempo en la que se presenta la infestación de la broca es mucho más rápida que la escala de tiempo en la que se presentan nuevas plantas o que desaparecen. El hecho de que la población sea constante implica que ésta es la suma del número de plantas susceptibles, infestadas y removidas o recuperadas.

6. La broca se puede propagar cuando de un cafeto infestado, o de sus frutos caídos infestados, la plaga se desplaza a una planta susceptible. Cabe resaltar que esta plaga se puede desplazar por varios medios, como por ejemplo los vuelos a plantas cercanas o en los recipientes donde se depositan los granos recogidos durante la cosecha, entre otros.

7. La investigación se implementará en una cosecha cualquiera.

\subsection{Variables y parámetros}

Las variables y parámetros del modelo son: $S(t), I(t)$ y $R(t)$, que representan el número de cafetos susceptibles a la broca, el número de cafetos infestados por la broca y el número de cafetos removidos o recuperados en el tiempo $t$ respectivamente. $N$ es el número de plantas en el cafetal de estudio, donde éste equivale a la suma de las tres poblaciones. $\beta$ y $\alpha$ son la tasa de infestación del cafetal y la tasa de recuperación de una planta de café debido a algún control respectivamente.

\subsection{Sistema de ecuaciones del modelo}

El sistema de ecuaciones diferenciales ordinarias que describe la dinámica del modelo es:

$$
\left\{\begin{array}{l}
\dot{S}=-\beta S I \\
\dot{I}=\beta S I-\alpha I \\
\dot{R}=\alpha I .
\end{array}\right.
$$

donde se considera una población inicial de susceptibles $S(0)=S_{0}$, una población inicial de infestados $I(0)=I_{0}$, y no se supone plantas de café recuperadas, es decir, $R(0)=0$.

Además, como la población total de plantas es constante, es decir, $N=$ $S+I+R$, entonces, la población recuperada se puede expresar en términos de las otras poblaciones $R=N-S-I$. 
El sistema (1) es desacoplado en una vía, es decir, en éste las dos primeras ecuaciones no dependen de la tercera, por tal razón se trabajará con el siguiente sistema:

$$
\left\{\begin{array}{l}
\dot{S}=-\beta S I \\
\dot{I}=\beta S I-\alpha I
\end{array}\right.
$$

Para determinar los puntos de equilibrio del sistema de ecuaciones que describe la dinámica y, asumiendo que el sistema de ecuaciones (1) es desacoplado en una vía, se analiza:

$$
\left\{\begin{array}{lll}
-\beta S I & = & 0 \\
\beta S I-\alpha I & = & 0
\end{array}\right.
$$

Si se asume que $S=0$ en la primera ecuación de (3), reemplazando este valor en la segunda ecuación se obtiene que $I=0$, luego el equilibrio es $(0,0)$.

De la segunda ecuación de (3), $I(\beta S-\alpha)=0$. Luego $I=0$ o $\beta S-\alpha=$ 0 . Si $I=0, S$ puede asumir cualquier valor. De esta manera se genera una semirecta de equilibrios y estos tienen la forma $(S, 0)$.

\section{Estimación del parámetro $\beta$}

Para estimar el parámetro $\beta$ que representa la tasa de infestación de un árbol de café, es necesario determinar la relación que existe entre las variables susceptibles $(S)$ e infestados $(I)$, es decir, encontrar la solución de la variable $I$ en términos de los parámetros y de la variable $S$. Para esto se elimina la variable temporal $t$, haciendo uso del sistema (2), así:

$$
\frac{d I}{d S}=\frac{\beta S I-\alpha I}{-\beta S I}=-1+\frac{\alpha}{\beta S} .
$$

Aplicando el método de separación de variables a la ecuación anterior, la solución general es dada por:

$$
I(S)=-S+\frac{\alpha}{\beta} \ln S+C,
$$

al usar las condiciones iniciales, es posible determinar la constante de integración

$$
C=N-\frac{\alpha}{\beta} \ln S_{0}
$$

ahora, reemplazando (6) en (5) se tiene la solución particular:

$$
I(S)=N-S+\frac{\alpha}{\beta} \ln \frac{S}{S_{0}} \operatorname{con} S_{0} \neq 0 .
$$


Otra manera de expresar la ecuación (5) que describe las trayectorias del sistema, es en términos de una nueva función $V_{i}$ que depende de $S$ e $I$ y es dada por:

$$
V(S, I)=I+S-\frac{\alpha}{\beta} \ln S=C .
$$

Obsérvese que para cada selección de una constante, $V(S, I)=C$ describe implícitamente una órbita o trayectoria del sistema. Ahora, dadas las condiciones iniciales del sistema, es decir, población inicial de susceptibles e infesta$\operatorname{dos} S(0)=S_{0}, I(0)=I_{0}$ respectivamente, se tiene:

$$
V\left(S_{0}, I_{0}\right)=I_{0}+S_{0}-\frac{\alpha}{\beta} \ln S_{0} .
$$

Por otro lado, si $t$ crece sin límite en (8) y definiendo a $S^{\infty}=\lim _{t \rightarrow \infty} S(t)$ e $I^{\infty}=\lim _{t \rightarrow \infty} I(t)$, se tiene:

$$
\lim _{t \rightarrow \infty} V(S, I)=\lim _{t \rightarrow \infty}\left(I(t)+S(t)-\frac{\alpha}{\beta} \ln S(t)\right)=V\left(S^{\infty}, I^{\infty}\right),
$$

donde $\lim _{t \rightarrow \infty} I(t)=0$, esto se tiene, ya que si sumamos las ecuaciones del sistema (3) se tiene que $(S+I)^{\prime}=-\alpha I$. Así $S+I$ es una función no negativa decreciente y por lo tanto tiene límite cuando $t \rightarrow \infty$. Además, la derivada de una función no negativa, suave y decreciente tiende a cero, lo que justifica que $\lim _{t \rightarrow \infty} I(t)=0$. Entonces,

$$
V\left(S^{\infty}, I^{\infty}\right)=V\left(S^{\infty}, 0\right)=S^{\infty}-\frac{\alpha}{\beta} \ln S^{\infty} .
$$

Debido a que la constante $C$ es única una vez dadas las condiciones iniciales $\left(S_{0}, I_{0}\right)$ de $S$ e $I$ respectivamente, entonces se satisface la relación (veáse [3] pág. 288):

$$
V\left(S_{0}, I_{0}\right)=V\left(S^{\infty}, 0\right)
$$

es decir,

$$
I_{0}+S_{0}-\frac{\alpha}{\beta} \ln S_{0}=S^{\infty}-\frac{\alpha}{\beta} \ln S^{\infty}
$$

Realizando algunos cálculos en la expresión anterior, la tasa de infestación de un árbol de café es expresada en términos de algunos de los parámetros del sistema de la siguiente manera:

$$
\beta=\frac{\alpha \ln \frac{S_{0}}{S^{\infty}}}{N-S^{\infty}} .
$$

donde $S_{0}$ es la población susceptible al inicio de la cosecha y $S^{\infty}$ representa la población susceptible al final de la cosecha. 
Es importante resaltar la relación indirecta que existe entre el parámetro estimado dado en la ecuación (9) con el umbral de infestación establecido en los supuestos del modelo (5\%), la cual indica que si se asume un umbral de infestación más alto entonces la subpoblación de infestados disminuye (esto se debe a que es más difícil cumplir la condición del umbral dado y la tasa de infestación se hace más pequeña). Puesto que la población total es constante, el número de plantas susceptibles iniciales aumenta. Como $\beta$ en (9) depende de $S_{0}$ y la función logaritmo natural es creciente, se tiene que la tasa de infestación de un árbol de café $\beta$ aumenta también.

\section{Análisis cualitativo del modelo}

\subsection{Análisis de las variables de estado}

Uno de los objetivos de esta sección es determinar una expresión para el número máximo de plantas de café infestadas. Para ésto se iguala a cero la derivada (4) y se obtiene el número crítico $S=\frac{\alpha}{\beta}$ para

$$
I(S)=N-S+\frac{\alpha}{\beta} \ln \frac{S}{S_{0}} \operatorname{con} S_{0} \neq 0 .
$$

Ahora, si $S<\frac{\alpha}{\beta}$ entonces $\frac{d I}{d S}>0$, es decir, $I(S)$ es creciente. Por otro lado, si $S>\frac{\alpha}{\beta}$ entonces $\frac{d I}{d S}<0$, luego $I(S)$ es decreciente. Por lo tanto, el número máximo de plantas infestadas denotado por $I_{M A X}$ ocurre cuando $S=\frac{\alpha}{\beta}$; y si se reemplaza esta expresión en la ecuación (10), se obtiene

$$
I_{M A X}=N-\frac{\alpha}{\beta}+\frac{\alpha}{\beta}\left[\ln \frac{\alpha}{\beta}-\ln S_{0}\right] .
$$

El análisis hecho para la función $I(S)$, se puede visualizar en la Figura 1, con una subpoblación de árboles susceptibles inicial $S_{0}=999$. La interpretación de ésta debe realizarse de derecha a izquierda, donde el número de árboles susceptibles se grafican a lo largo del eje horizontal y, los infestados en el eje vertical. Obsérvese en la Figura 1, que la dinámica para la subpoblación de árboles susceptibles inicia en 999 y la de infestados en 1, como ésta se debe interpretar en sentido antihorario entonces a medida que $S$ disminuye, $I(S)$ aumenta hasta alcanzar el valor máximo $I_{M A X}$. Luego, la función decrece. Por otro lado, la Figura 2 muestra la evolución en el tiempo de las variables de estado, la curva azul representa las plantas de café susceptibles, la verde infestadas y por último, la roja la población de plantas de café recuperadas. Además usando las dos primeras ecuaciones del sistema (1) se realiza el siguiente análisis: 


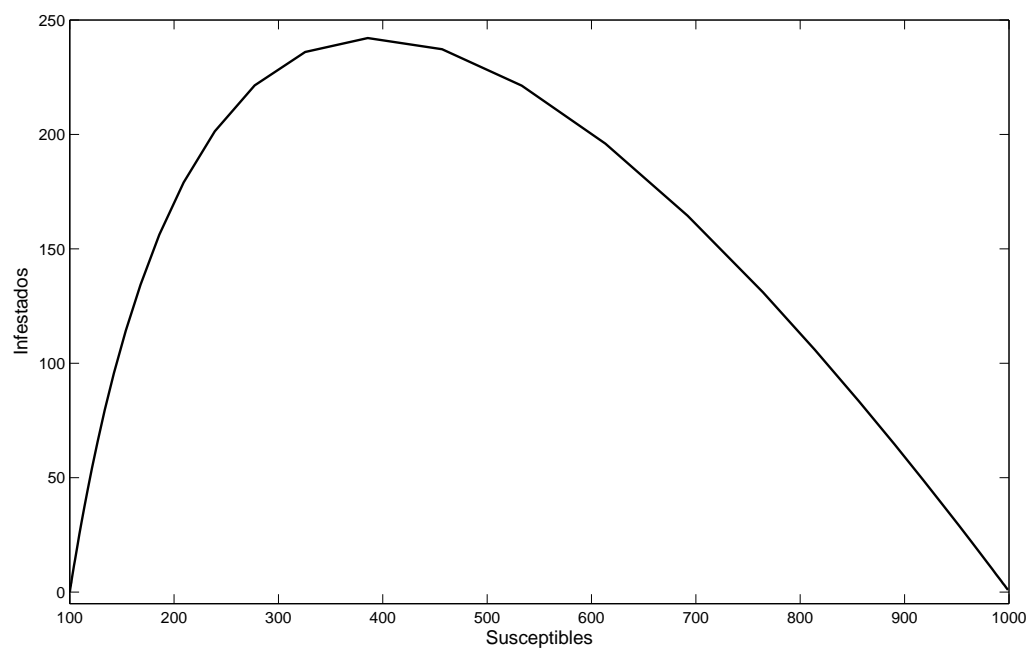

Figura 1: Trayectoria que describe la ecuación (10) referente a las poblaciones de susceptibles vs infestados, con condición inicial $S_{0}=999$.

- De la primera ecuación, se cumple que $\frac{d S}{d t}<0$ dado que $\beta, S$ e $I$ son positivos para todo $t$, luego el número de cafetos susceptibles $S$ decrece durante toda la dinámica, como lo ilustra la curva azul en la Figura 2 , donde inicialmente, se tomó como caso de estudio a 999 árboles susceptibles y al final de la curva solo hay 100 .

- De la segunda ecuación del sistema (1), si $S>\frac{\alpha}{\beta}$ (para este caso particular $S>391.0349$ ) entonces $\frac{d I}{d t}>0$, luego $I$ crece hasta un punto máximo $I \max =242.1908$. Esto puede ser observado en la Figura (2) curva azul. Además si $S<\frac{\alpha}{\beta}(S<391.0349)$ entonces $\frac{d I}{d t}<0$, por tanto $I$ decrece hasta el valor mínimo (Ver Fig. 2).

- De la tercera ecuación del sistema (1), siempre se tiene que $\frac{d R}{d t}>0$, luego la población de plantas recuperadas crece debido al constante y efectivo control descrito por el parámetro $\alpha$ como lo ilustra la curva roja.

\subsection{Simulaciones numéricas}

Con el objetivo de visualizar y análizar mejor la dinámica del sistema (1), se hace una serie de simulaciones en el tiempo en ambiente Matlab, con datos hipotéticos. Para ésto se iniciará variando el parámetro $\alpha$ que representa la tasa de 
Los parámetros utilizados para la realización de las simulaciones numéricas se muestran en la Tabla 1 .

\begin{tabular}{l|c}
\hline \hline Parámetro & Valor \\
\hline Número de cafetos susceptibles a la broca inicialmente & $S(0)=999$ \\
Número de cafetos infestados por la broca inicialmente & $I(0)=1$ \\
Número de cafetos removidos inicialmente & $R(0)=0$ \\
Número de plantas en el cafetal de estudio & $N=1000$ \\
\hline \hline
\end{tabular}

Tabla 1: Parámetros del Modelo.

En las figuras dadas a continuación, la curva de color azul representa la población de plantas de café susceptibles, la verde los infestados y la roja los recuperados.

\section{Escenario 1.}

Para la simulación de este escenario, se utilizan los valores de la Tabla 1 , además se asume el valor para la tasa de recuperación de una planta de café igual a uno $(\alpha=1)$. Para encontrar la tasa de infestación de la dinámica, se utiliza la ecuación (9), luego $\beta=0,0026$. Con lo anterior se obtiene la Figura 3 .

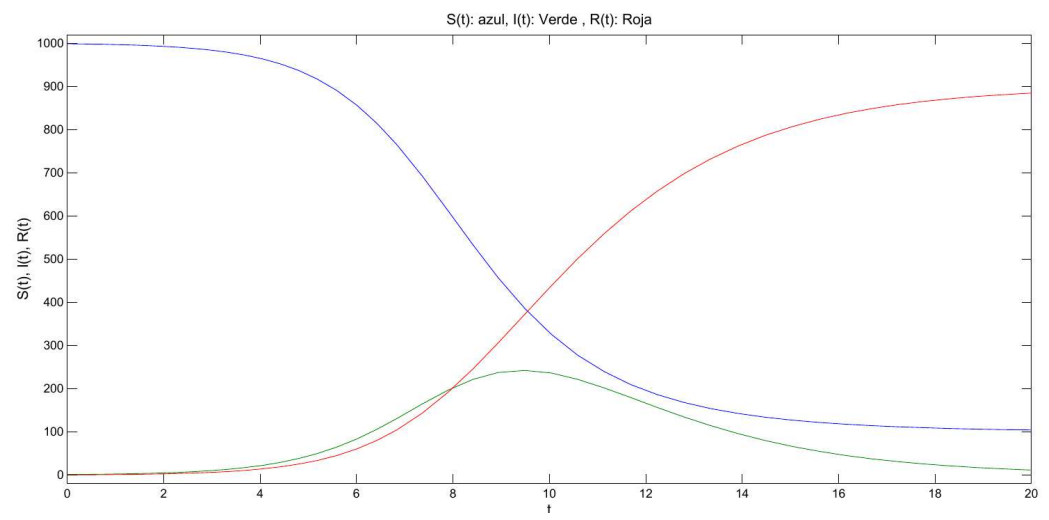

Figura 3: Dinámica del sistema para las variables de estado con $\alpha=1$. La curva azul representa la población de plantas susceptibles a la broca, la línea verde las infestadas y la roja representa las plantas recuperadas. 


\section{Escenario 2.}

En este escenario, se utilizan los valores de la Tabla $1, \alpha=0.3$ y $\beta=4,2451 \times$ $10^{-5}$. Con éstos se obtiene la Figura 4

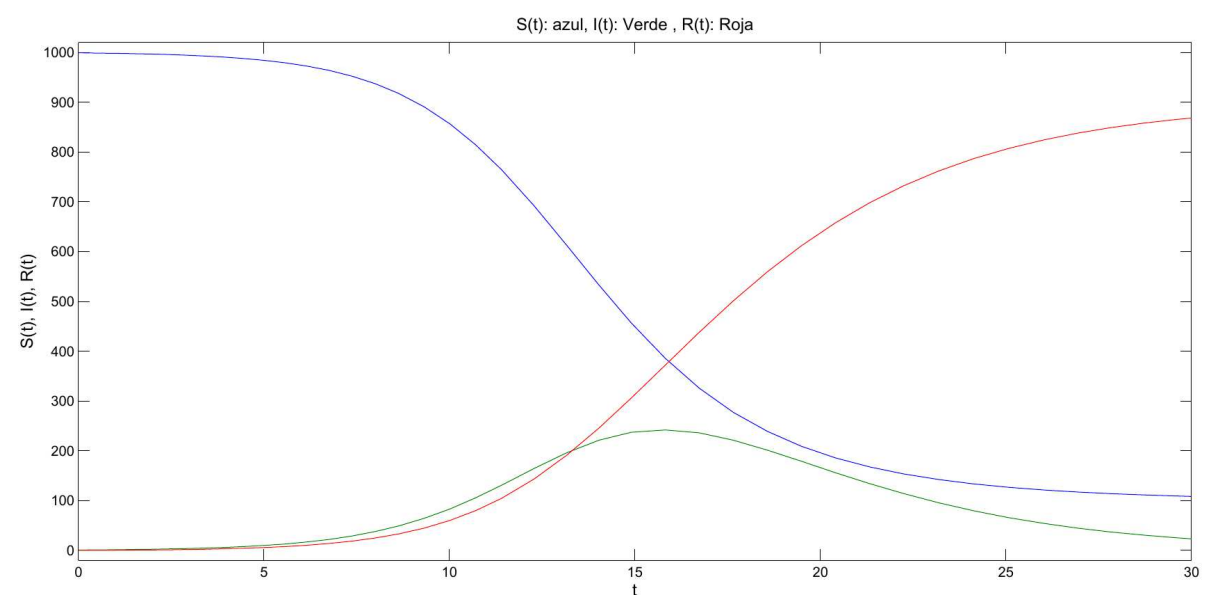

Figura 4: Dinámica del sistema para las variables de estado con $\alpha=0.3$.

En las Figuras 3 y 4, la dinámica de árboles susceptibles siempre tiende a disminuir, sin importar la tasa de recuperación, esto ocurre dado que la broca ha infestado dicha población, esto se observa en curva azul. Debido a este hecho se dispara la cantidad de cafetos infestados como lo ilustra la curva verde, pero este comportamiento es interrumpido dado que se aplica un control en ambos escenarios.

Para obtener el comportamiento mostrado en la Figura 3 se utilizó una tasa de recuperación $\alpha=1$, a su vez para la Figura $4 \alpha=0.3$. Se aprecia que la población de recuperados, curva roja en la Figura 3 , crece más rápido que en la Figura 4, debido a que la tasa de recuperación es mayor en el primer escenario que en el segundo. Para la población de plantas infestadas, se tiene que a mayor valor del parámetro $\alpha$, el número máximo de plantas infestadas, $I_{M A X}$ se alcanza en un menor tiempo.

\section{Escenario 3.}

Debido a la importancia que tiene el parámetro $\alpha$ y la relación directa con el parámetro estimado $\beta$ como lo ilustra la ecuación (9), se realizó la Figura 5, en ésta se simulan 10 escenarios diferentes con el objetivo de observar la dinámica del sistema (1) cuando dichos parámetros cambian. Para esto se utilizó una distribución uniforme la cual permite que el parámetro $\alpha$ tome valores aleatorios en el intervalo $[0,1]$. 

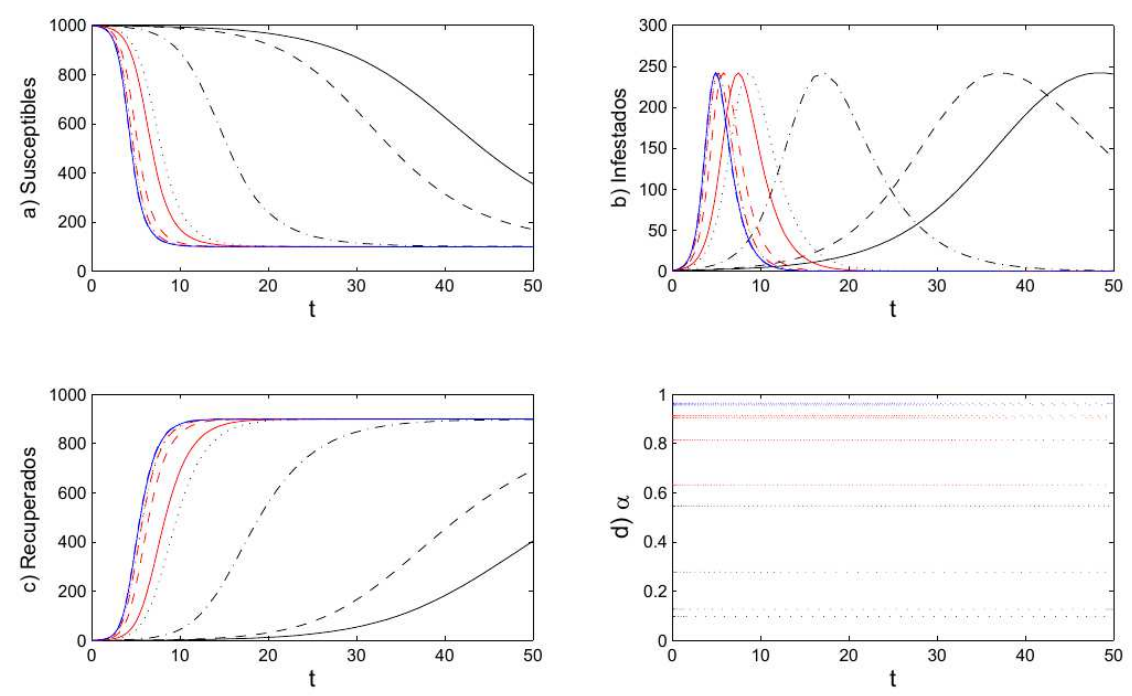

Figura 5: Dinámica para las distintas poblaciones con relación al tiempo. En este caso se varía el parámetro $\alpha$ en el intervalo $[0,1]$.

La Figura 5 está compuesta por cuatro subgráficas, donde se muestra el comportamiento de cada subpoblación de plantas de café $a$ ) susceptibles, $b$ ) infestadas y $c$ ) recuperadas además en la cuarta subgráfica $d$ ), se muestra los valores que toma el parámetro $\alpha$ donde para cada dato se le fue asignado un color o forma diferente, la cual coincide con las otras tres subgráficas.

Para todos los distintos valores de $\alpha$, el número máximo de infestados $I_{M A X}$ es el mismo y eso se debe a la forma de la ecuación (11). Este hecho se evidencia en la subgráfica $b$ ).

Cuando el parámetro $\alpha$ toma valores por debajo de 0.5 , lo cual se muestra en la subgráfica $d$ ) de la Figura 5, se aprecia en la subgráfica b) que la cantidad de árboles de café infestados por la broca perdura casi durante todo el tiempo que dura la cosecha y se evidencia además que el $I_{M A X}$ tarda más tiempo en alcanzarse, a diferencia cuando $\alpha$ toma valores mayores que 0.5 , es decir, si la tasa de recuperación $\alpha$ supera 0.5 , el número de cafetos infestados por la broca se dispara hasta alcanzar su valor máximo con mayor rapidez.

También cuando $\alpha$ toma valores inferiores a 0.5 , la subpoblación de plantas susceptibles decrece más lentamente, a diferencia de cuando toma valores mayores. Además si $\alpha<0.5$, las plantas de café tardan más tiempo en recuperarse. 


\section{Escenario 4.}

De la ecuación (9), puede obtenerse una expresión para $\frac{\alpha}{\beta}$, la cual depende del parámetro $S^{\infty}$, que representa la población susceptible al final de la cosecha, para una población fija $N$ y $S_{0}$. Debido a la importancia que tiene el valor $S^{\infty}$, se realizaron las Figuras 6 y 7 En éstas se simulan 6 dinámicas diferentes con el objetivo de observar la influencia de $S^{\infty}$ en el comportamiento del sistema (11). Para esto se utilizó una distribución uniforme la cual permite que $S^{\infty}$ tome valores aleatorios en el intervalo [0,300].
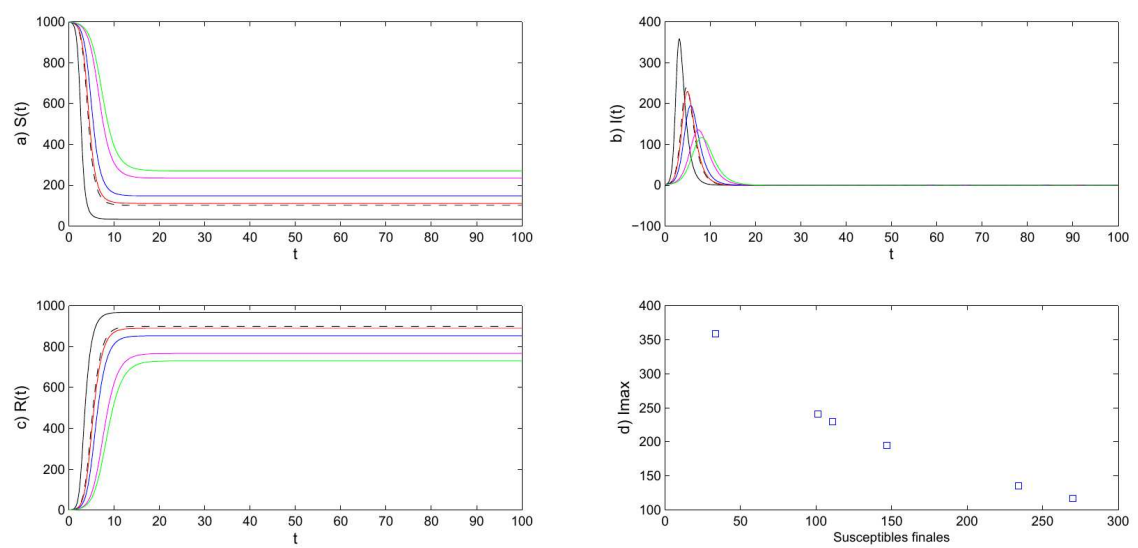

Figura 6: Comportamiento en el tiempo de las subpoblaciones de plantas susceptibles, infestadas y recuperadas.

La Figura 6 muestra el comportamiento en el tiempo de las subpoblaciones que intervienen en la dinámica, además del número final de plantas susceptibles. Se puede ver aquí, por ejemplo, la línea continua de color negro en las tres primeras subgráficas a), b), c). En a) se observa que el número de plantas susceptibles $S(t)$ decrece rápidamente hasta estabilizarse en el valor $S^{\infty}=33$, como lo muestra la Tabla (2). Para la subgrafica b), la población del número de plantas infestadas $I(t)$ crece muy rápido hasta llegar al número máximo $I_{M A X}=358$, luego decrece. Ahora, en la subgráfica c) las curvas que representan el número de plantas recuperadas $R(t)$ siempre crecen. Por último, la subgráfica d) es una reprentación de la Tabla 2 , donde el eje horizontal representa el número final de susceptibles y en el eje vertical el número máximo de infestados por simulación. La Figura 7, esta dividida en cuatro subgráficas a), b), c), d). En a) superior izquierda representa la dinámica del porcentaje de los infestados en el eje vertical y el porcentaje de los susceptibles en el eje horizontal. Recuerde que estas trayectorias deben leerse en sentido antihorario. Obsérvese la línea roja, esta curva se obtuvo cuando la población final de susceptibles es de un $13 \%$ ver 


\begin{tabular}{c|cc}
\hline \hline Susceptibles finales & $\mathrm{I}_{\max }$ & Color de la curva \\
\hline 33 & 358 & Negra continua \\
101 & 240 & Negra discontinua \\
110 & 229 & Roja \\
146 & 195 & Azul \\
234 & 135 & Morada \\
270 & 117 & Verde \\
\hline \hline
\end{tabular}

Tabla 2: Número máximo de plantas infestadas con relación al número de susceptibles al final de la cosecha.

\begin{tabular}{c|cll}
\hline \hline Susceptibles finales $\%$ & $I_{\max } \%$ & $\beta \%$ & Color de la curva \\
\hline 1 & 45 & 0.47 & Negra continua \\
2 & 43 & 0.43 & Negra discontinua \\
5 & 31 & 0.31 & Roja \\
8 & 26 & 0.27 & Azul \\
19 & 15 & 0.20 & Morada \\
21 & 14 & 0.198 & Verde \\
\hline \hline
\end{tabular}

Tabla 3: Porcentaje de: plantas susceptibles finales, número máximo de infestación y la tasa de infestación del cafetal.

Tabla 3, la trayectoria inicia con un $99 \%$ de población susceptible y un $1 \%$ de población infestada. Además alcanza un porcentaje máximo de infestación del $31 \%$. Nuevamente, usando la Tabla 3, la tasa de infestación del cafetal para la curva roja es de $0.31 \%$, ver también la subgráfica b).

En c) de la Figura 7, se muestra el comportamiento en porcentajes de la suma del número de plantas susceptibles $S(t)$ y recuperadas $R(t)$ en el eje vertical, y en eje horizontal el tiempo. En d) de la Figura 7, se gráfica el comportamiento del porcentaje de la población de infestados respecto al tiempo.

Por ejemplo, mientras que la curva azul en la subgráfica c) aumenta en d) decrece, este comportamiento se debe a que la población de plantas de café se esta considerando constante por lo tanto la suma de los tres poncentajes de las subpoblaciones $S(t) \%, I(t) \%, R(t) \%$ debe dar el $100 \%$. 

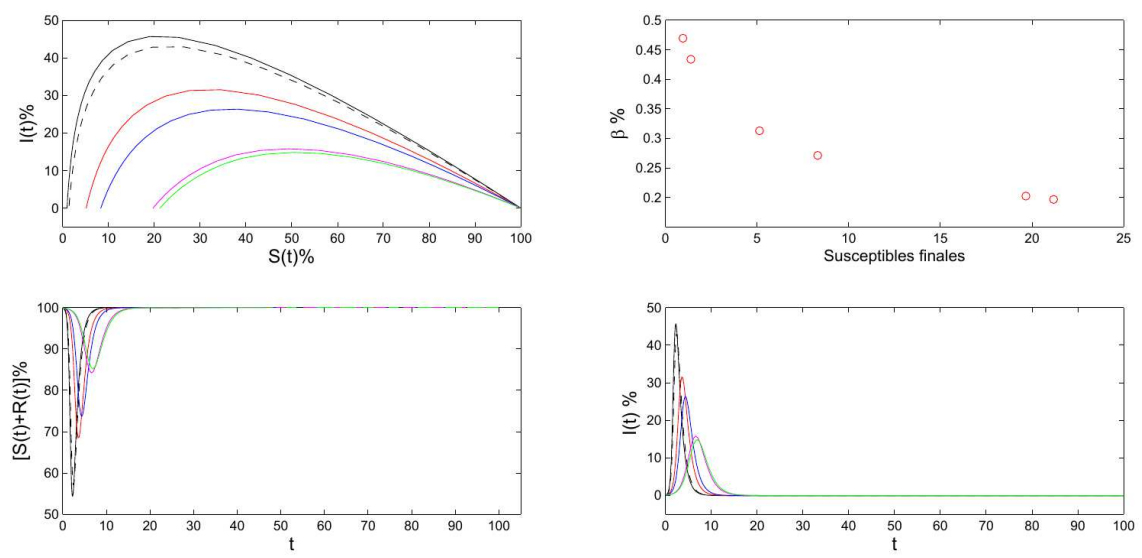

Figura 7: Trayectorias que describen el porcentaje de infestación de las subpoblaciones.

\section{Conclusiones}

Los diferentes valores de la tasa de recuperación son atribuibles a los variados mecanismos de control de la broca como son: el control manual, control biológico con el hongo Beauveria bassiana y las avispas Cephalonomia stephanoderis, el control químico y el zoqueo de cafetales.

La dinámica de árboles susceptibles siempre tiende a disminuir, sin importar el valor que tome alpha, la tasa de recuperación, esto ocurre dado que la broca ha infestado el cafetal en estudio.

Para cualquier valor de la tasa de recuperación de la broca $\alpha$, siempre existirá un día durante el desarrollo de los frutos donde la cantidad de cafetos infestados por la broca alcanzará su nivel máximo, después de ese día la cantidad de cafetos afectados por la broca disminuirá notablemente.

Para todos los distintos valores de la tasa de recuperación $\alpha$, el número máximo de infestados $I_{M A X}$ es el mismo y eso se debe a la forma de la ecuación (11). Este hecho se evidencia en la subgráfica b).

La dinámica conjunta del sistema (1), no se ve afectada de una manera drástica, es decir no hay un cambio topológico, al variar la tasa de recuperación $(\alpha)$, ésto indica que el sistema no es sensible a los cambios pequeños de este parámetro. 


\section{Referencias}

[1] Arcila, P.; Jaramillo, R.; Baldión, R.; Bustillo, A.E. (1993) "La floración del cafeto y su relación con el control de la broca", Avances Técnicos Cenicafé 193: 1-6.

[2] Bergamin, J. (1943) "Contribução para o conhecimento da biologia da broca-do-café Hypotenemus hampei (Ferrari, 1867) (Coleoptera: Ipidae)", Arquivos do Instituto Biologico 14: 31-72.

[3] Brauer, F.; Castillo-Chavez, C. (2001) Mathematical Models in Population Biology and Epidemiology, 2nd Ed. Springer, New York.

[4] Bustillo, A.E. (1991) "Perspectivas de manejo integrado de la broca del café, Hypotenemus hampei en Colombia", Agricultura Tropical (Colombia) 28(1): 83-93.

[5] Bustillo, A.E. (2007) El manejo de cafetales y su relación con el control de la broca del café en Colombia. Boletín Técnico, Cenicafé, Chinchiná, Caldas, Colombia.

[6] Bustillo, A.E.; Villalba, D.A. (2004) "Efecto del clima y condiciones del cultivo del café en la biología y comportamiento de la broca del café, $H y$ pothenemus hampei (Ferrari) (Coleoptera: Curculionidae: Scolytinae)", en: Anais Workshop Internacional Manejo da Broca do Café, Londrina, Brasil: 37-50.

[7] Camayo, V.; Arcila, P. (1997) "Desarrollo floral del cafeto en condiciones de la zona cafetera colombiana (Chinchiná-Caldas)", Avances Técnicos Cenicafé 245: 1-8.

[8] Jaramillo, A.; Guzmán, O. (1984) "Relación entre la temperatura y crecimiento en coffea arabiga L., variedad caturra”, Cenicafé 35(3): 57-65.

[9] Mejía, P. (1998) Manejo Integrado de la Broca del Café. Federación Nacional de Cafeteros de Colombia, Centro Nacional de Investigaciones de Café, Colombia.

[10] Miguel, A.E.; Paulini, A.E. (1975) "Velocidade de penetraçao da broca do café Hypotenemus hampei (Ferrari) no fruto do café", en: Resumos. Congresso Brasileiro de Pesquisas Cafeeiras, 3. Curitiba: 8-21.

[11] Perko, L. (1996) Differential Equations and Dynamical Systems, 2nd Edition. Springer, New York. 
[12] Ruiz, C.R. (1996) Efecto de la Fenología del Fruto del Café sobre los Parámetros de la Tabla de Vida de la Broca del Café; Hypothenemus hampei (Ferrari). Tesis de Ingeniero Agrónomo, Facultad de Ciencias Agropecuarias, Universidad de Caldas, Manizales, Colombia.

[13] UCR (1995) Control Biológico de la Broca del Café. Universidad de Costa Rica, San José.

[14] Vélez, B.E.; Jaramillo, A.; Chaves, B.; Franco, M. (2000) “Distribución de la floración y cosecha de café en tres altitudes", Avances Técnicos Cenicafé 272: $1-4$.

[15] Wood, D.L. (1982) "The role of pheromones, kairomones and allomones in the host selection and colonization behavior of bark beetles", Annual Review of Entomology 27: 411-446. 